\title{
A comparison between heart rate and heart rate variability as indicators of cardiac health and fitness
}

\author{
Catharina C. Grant ${ }^{1}{ }^{*}$, Carien Murray ${ }^{1}$, Dina C. Janse van Rensburg ${ }^{1}$ and Lizelle Fletcher ${ }^{2}$ \\ ${ }^{1}$ Section Sports Medicine, University of Pretoria, Pretoria, South Africa \\ ${ }^{2}$ Department of Statistics, University of Pretoria, Pretoria, South Africa
}

\section{Edited by:}

Jerzy Sacha, Regional Medical

Center, Poland

Reviewed by:

Jerzy Sacha, Regional Medical

Center, Poland

George E. Billman, The Ohio State

University, USA

*Correspondence:

Catharina C. Grant, Section Sports Medicine, University of Pretoria, P.O.

Box 37897, Faerie Glen 0043,

Gauteng, Pretoria, South Africa

e-mail:rina.grant@up.ac.za
Quantification of cardiac autonomic activity and control via heart rate (HR) and heart rate variability (HRV) is known to provide prognostic information in clinical populations. Issues with regard to standardization and interpretation of HRV data make the use of the more easily accessible HR on its own as an indicator of autonomic cardiac control very appealing. The aim of this study was to investigate the strength of associations between an important cardio vascular health metric such as $\mathrm{VO}_{2}$ max and the following: $\mathrm{HR}, \mathrm{HRV}$ indicators, and HR normalized HRV indicators. A cross sectional descriptive study was done including 145 healthy volunteers aged between 18 and 22 years. HRV was quantified by time domain, frequency domain and Poincaré plot analysis. Indirect $\mathrm{VO}_{2}$ max was determined using the Multistage Coopers test. The Pearson correlation coefficient was calculated to quantify the strength of the associations. Both simple linear and multiple stepwise regressions were performed to be able to discriminate between the role of the individual indicators as well as their combined association with $\mathrm{VO}_{2} \max$. Only $\mathrm{HR}$, RR interval, and pNN50 showed significant ( $p<0.01, p<0.01$, and $p=0.03$ ) correlations with $\mathrm{VO}_{2}$ max. Stepwise multiple regression indicated that, when combining all $\mathrm{HRV}$ indicators the most important predictor of cardio vascular fitness as represented by $\mathrm{VO}_{2} \max$, is HR. HR explains $17 \%$ of the variation, while the inclusion of HF (high frequency HRV indicator) added only an additional $3.1 \%$ to the coefficient of determination. Results also showed when testing the normalized indicators, $\mathrm{HR}$ explained of the largest percentage of the changes in $\mathrm{VO}_{2}$ max $(16.5 \%)$. Thus, HR on its own is the most important predictor of changes in an important cardiac health metric such as $\mathrm{VO}_{2}$ max. These results may indicate that during investigation of exercise ability $\left(\mathrm{VO}_{2}\right.$ max $)$ phenomena, quantification of HRV may not add significant value.

Keywords: autonomic cardiac control, prognostic indicators, exercise ability

\section{INTRODUCTION}

It is known that cardiovascular disease (CVD) has reached pandemic proportions worldwide. It is identified as one of the most important causes of death in several countries including USA, Pacific and Middle Eastern countries, China and Europe (Barakat et al., 2012; Go et al., 2013). Factors, contributing to this pandemic is: smoking, physical activity/fitness, blood pressure, blood glucose, total cholesterol levels, weight, and diet. Of these factors, also called cardio vascular health metrics (Yang et al., 2012), the level of activity and fitness is a relative easy way to modify a person's CVD risk factor. However, low physical activity levels is also one of the most common problems identified across different age group contributing toward the CVD pandemic (Barakat et al., 2012). It is known that an increase in fitness/exercise capacity measured by $\mathrm{VO}_{2} \max$, results in a reduction in CVD risk (Barakat et al., 2012). Active individuals present half the coronary artery disease risk compared to a very inactive group. Exercise and increased fitness not only help to prevent disease risk factors but can also treat existing risk factors such as high insulin levels, hypertension, hyperlipidaemia and obesity.
The link between cardiac autonomic neuropathy (increased sympathetic, decreased parasympathetic activity or a combination of the two), and CVD is well established (Lahiri et al., 2008). Quantification of cardiac autonomic activity and control via heart rate (HR) and heart rate variability (HRV) is known to provide prognostic information in clinical populations. HR is used as a CV mortality risk factor indicator and HRV as a predictor of disease outcome (Kannel et al., 1987; Bravi et al., 2011; Sacha et al., 2013).

Quantification of HRV, integrating the sympathetic and parasympathetic cardiac influences, is a complex measurement of the autonomic nervous system and its responses to internal and external stimuli (Lahiri et al., 2008). Issues with regards to standardization and interpretation (Grant et al., 2011) of HRV data make the use of the more easily accessible HR on its own as an indicator of autonomic cardiac control very appealing. It is important to establish if the more complex concept of HRV determination adds prognostic value above HR measurements. In an effort to quantify the contribution of HR to the prognostic value of HRV, the aim of this study was to investigate the strength of associations between an important cardio vascular health metric 
such as $\mathrm{VO}_{2}$ max and $\mathrm{HR}$, as well as between $\mathrm{VO}_{2}$ max and $\mathrm{HRV}$ indicators including RMSSD, pNN50, LF, HF, LF/HF, SD1, and SD2 (abbreviations explained in Table 1). HR normalized HRV indicators were also calculated and used as regressors to determine if $\mathrm{HR}$ independent variability shows similar associations with $\mathrm{VO}_{2}$ max than standard HRV indicators. Regression analyses were employed to determine the relative importance of $\mathrm{HR}$ and $\mathrm{HRV}$ indicators as predictors of an important cardio vascular health metric such as $\mathrm{VO}_{2}$ max.

The primary hypothesis was that $\mathrm{HR}$ and $\mathrm{RR}$ intervals show stronger correlations with $\mathrm{VO}_{2}$ max than the $\mathrm{HRV}$ indicators, and are on their own stronger predictors of $\mathrm{VO}_{2} \max$ than the more complex indicators of HRV (RMSSD, pNN50, LF, HF, LF/HF, $\mathrm{SD} 1$, and SD2). A secondary hypothesis was that, when including HR and HR normalized indicators of variability (RMSSD/RR, $\left.\mathrm{LF} / \mathrm{RR}^{2}, \mathrm{HF} / \mathrm{RR}^{2}, \mathrm{LF} / \mathrm{HF}, \mathrm{SD} 1 / \mathrm{RR}, \mathrm{SD} 2 / \mathrm{RR}\right)$ regression analysis will show that $\mathrm{HR}$ is still the most important predictor of $\mathrm{VO}_{2} \max$.

\section{MATERIALS AND METHODS BACKGROUND AND DETERMINATION OF THE HEALTH METRIC $\mathrm{VO}_{2}$ MAX}

The gold standard in fitness testing is generally regarded as the $\mathrm{VO}_{2}$ max test for measuring aerobic fitness or cardiorespiratory endurance. $\mathrm{VO}_{2}$ max is defined as the maximum volume of oxygen that can be utilized in $1 \mathrm{~min}$ during maximal exercise. It is measured in millilitres of oxygen used in 1 min per kilogram of body weight, ( $\mathrm{ml} / \mathrm{kg} / \mathrm{min}$ ) (Wilmore and Costill, 2004). Elite endurance athletes are known to have high $\mathrm{VO}_{2}$ max values and it is thought that the more oxygen the body can use during strenuous exercise, the more energy it can produce (Wilmore and Costill, 2004).

$\mathrm{VO}_{2}$ max can be measured directly or indirectly. The direct measurement needs to be done in a laboratory and is expensive, time consuming and requires special expertise. The test subject must reach his/her maximum work capacity in order to determine $\mathrm{VO} 2$ max accurately. The test is done on a treadmill or a bicycle and a strict protocol is followed. The athlete is required to exercise while the speed and intensity is gradually increased. The

Table 1 | HRV indicators defined.

\begin{tabular}{|c|c|}
\hline HRV indicator & Definition \\
\hline Mean RR (s) & $\begin{array}{l}\text { The mean of the intervals between successive ORS } \\
\text { complexes }\end{array}$ \\
\hline RMSSD (ms) & $\begin{array}{l}\text { Root mean square of the standard deviation between } \\
\text { RR intervals }\end{array}$ \\
\hline pNN50 (\%) & $\begin{array}{l}\text { The percentage of successive RR interval differences } \\
\text { over the entire measurement larger than } 50 \mathrm{~ms}\end{array}$ \\
\hline LF Power $\left(\mathrm{ms}^{2}\right)$ & Peak between 0.04 and $0.15 \mathrm{~Hz}$ \\
\hline HF Power $\left(\mathrm{ms}^{2}\right)$ & Peak between 0.15 and $0.5 \mathrm{~Hz}$ \\
\hline $\mathrm{LF} / \mathrm{HF}$ & LF Power $\left(m s^{2}\right)$ divided by HF Power $\left(\mathrm{ms}^{2}\right)$ \\
\hline SD1 (ms) & $\begin{array}{l}\text { Indicator of the standard deviation of the instantaneous } \\
\text { RR variability }\end{array}$ \\
\hline $\mathrm{SD} 2(\mathrm{~ms})$ & $\begin{array}{l}\text { Indicator of the standard deviation of the continuous or } \\
\text { long term variability of the heart rate }\end{array}$ \\
\hline
\end{tabular}

volume and oxygen concentration of the inhaled and exhaled air of the athlete is measured to determine how much oxygen is used. The oxygen consumption usually rises in direct relationship to the increase in exercise intensity up to a certain point. At this point the oxygen consumption reaches a plateau and does not rise further even with a further rise in intensity. This plateau marks the $\mathrm{VO}_{2}$ max.

$\mathrm{VO}_{2}$ max can also be determined indirectly, as an estimate of the true $\mathrm{VO}_{2}$ max. The Cooper $12 \mathrm{~min}$ run test was used in the current study. This assessment uses a set period of time (12 min) and scoring according to distance (in meters). The participants were asked to run or walk for $12 \mathrm{~min}$ as fast as possible (Cooper, 1968). This test was performed on a $400 \mathrm{~m}$ tartan athletics track. A warm-up of light aerobic activities and flexibility exercises was performed for $5 \mathrm{~min}$ before the start of the test. Testers were placed at $100 \mathrm{~m}$ intervals around the track as a source of verbal motivation. A prediction of $\mathrm{VO}_{2 \max }$ from the distance covered at the end of the $12 \mathrm{~min}$ period was obtained by applying the distance run to the Cooper regression equation: $\mathrm{VO}_{2} \max (\mathrm{ml} / \mathrm{kg} / \mathrm{min})=0.0268$ (distance covered in meters) 11.3 (Cooper, 1968).

The sedentary person has a much lower $\mathrm{VO}_{2}$ max generally compared to the active fit individual, with the elite endurance athlete having the highest $\mathrm{VO}_{2}$ max. Genetics play a role in an individual's exercise ability but $\mathrm{VO}_{2}$ max can be improved with physical training. The more unfit, the more the $\mathrm{VO}_{2} \max$ can be improved. As exercise capacity improves, skeletal muscle strength and endurance also improve (Paterson et al., 2004; Stringer, 2010).

\section{STUDY DESCRIPTION}

A hypotheses driven, cross sectional, descriptive study was performed. The study protocol was submitted and approved by the Ethics Committee of the University. A total of 145 healthy participants from a group of 235 volunteers were accepted to take part in the research study. Exclusion criteria included refusal to give voluntary written informed consent; a history of cardiovascular, hepatic, respiratory, or renal impairment, as well as pulmonary, metabolic, and orthopaedic diseases requiring medical attention; lung/respiratory tract infection in the previous 2 weeks; and medication that could influence cardiovascular control and psychological disorders. None of the participants were professional athletes or high-level sport participants. All participants gave written informed consent before commencement of the intervention. Participants fasted overnight and were asked not to use any caffeine, alcohol or to smoke $24 \mathrm{~h}$ prior to the measurements. Measurements were taken in a temperature regulated, quiet environment, in the morning before $12 \mathrm{H} 00$. The Polar 810E HR monitor system was used to record supine RR intervals for a $10 \mathrm{~min}$ period. The 5 to $10 \mathrm{~min}$ period of this recording was used to quantify the HRV. Computer software from the University of Kuopio, Finland calculated the HRV indicator values by time domain analysis (RR, STDRR, RMSSD, and pNN50), frequency domain analysis (LF, HF, and LF/HF), and Poincaré plot analysis (SD1 and SD2) (Mourot et al., 2004). HRV indicators determined are listed in Table 1; Mourot et al., 2004). 


\section{STATISTICAL ANALYSES}

The Pearson's correlation coefficient $(r)$ was calculated. Correlation strength were defined as very low if rho is smaller than 0.2 ; low to moderate if rho is larger than 0.2 but smaller than 0.4 ; and moderate if rho is larger than 0.4 but less than 0.6 (Landis and Koch, 1977). The level of significance was set at the conventional $5 \%$, thus, when $p$-values were less than 0.05 , the associations were identified as statistically significant.

To test the hypotheses, linear regression was used to determine the most important predictors of changes in cardio vascular fitness and exercise ability as represented by $\mathrm{VO}_{2}$ max. Indicators included were: HR, RR interval, RMSSD, pNN50, LF, HF, LF/HF, SD1, SD2. The following HR normalized HRV indicators were also included in a separate set of regression analyses: RMSSD/RR, $\mathrm{LF} / \mathrm{RR}^{2}, \mathrm{HF} / \mathrm{RR}^{2}, \mathrm{LF} / \mathrm{HF}, \mathrm{SD} 1 / \mathrm{RR}$, and SD2/RR. Both simple linear and multiple stepwise regressions were performed to be able to discriminate between the role of the individual indicators as well as their combined relationship with $\mathrm{VO}_{2} \max$ (Field, 1994).

\section{RESULTS}

A summary of the participants $(N=145)$ and HRV indicator values are displayed in Tables 2, 3 .

Table 2 | Median, mean and standard deviation (SD) of participant body mass index (BMI) and exercise capacity $\left(\mathrm{VO}_{2} \mathrm{max}\right)$.

\begin{tabular}{llll}
\hline & Mean & SD & Median \\
\hline $\mathrm{BMI}\left(\mathrm{kg} / \mathrm{m}^{2}\right)$ & 23.07 & 2.36 & 22.62 \\
$\mathrm{VO}_{2} \mathrm{max}(\mathrm{ml} / \mathrm{kg} / \mathrm{min})$ & 49.54 & 8.79 & 53.53 \\
$2.4 \mathrm{~km}$ run time $(\mathrm{s})$ & 733.71 & 179.10 & 661.50 \\
\hline
\end{tabular}

Table 3 | Mean, standard deviation (SD), median and inter quartile range of HRV indicators and normalized HRV indicators.

\begin{tabular}{lclll}
\hline & Mean & SD & Median & $\begin{array}{l}\text { Inter quartile } \\
\text { range(01;03) }\end{array}$ \\
& & & & $186.25(791.00 ; 977.25)$ \\
Mean RR & 894.10 & 137.03 & 884.50 & \\
(ms) & & & & \\
Mean HR & 69.22 & 10.5 & 68.78 & $14.61(61.83 ; 76.44)$ \\
(beats per & & & & \\
minute) & & & & \\
RMSSD (ms) & 76.04 & 44.84 & 65.00 & $53.25(43.40 ; 96.65)$ \\
pNN50 (\%) & 39.08 & 22.84 & 38.30 & $39.85(18.40 ; 59.25)$ \\
LF (ms ${ }^{2}$ ) & 1148.19 & 1291.74 & 999.00 & $1336(582.50 ; 1918.50)$ \\
HF (ms ${ }^{2}$ ) & 2760.15 & 3465.31 & 1721.00 & $2627(582.50 ; 1918.50$ \\
LF/HF & 0.81 & 0.58 & 0.67 & $0.74(0.35 ; 1.09)$ \\
SD1(ms) & 54.06 & 31.83 & 46.10 & $37.80(30.95 ; 68.75)$ \\
SD2(ms) & 90.48 & 37.17 & 83.10 & $51.60(64.00 ; 115.60)$ \\
NORMALIZED HRV INDICATORS & & \\
RMSSD/RR & 0.08 & 0.04 & 0.07 & $0.05(0.05 ; 0.10)$ \\
LF/RR ${ }^{2}$ & 0.0017 & 0.0013 & 0.0012 & $0.001(0.001 ; 0.002)$ \\
HF/RR ${ }^{2}$ & 0.0031 & 0.0034 & 0.0020 & $0.002(0.001 ; 0.003)$ \\
SD1/RR & 0.060 & 0.030 & 0.050 & $0.04(0.03 ; 0.07)$ \\
SD2/RR & 0.10 & 0.040 & 0.090 & $0.05(0.07 ; 0.12)$ \\
& & & &
\end{tabular}

The HR, RR intervals, and HRV indicators (normalized and non-normalized) were compared to the $\mathrm{VO}_{2} \max$ of participants results, to establish any significant relationships (Table 4).

The results shown in Table 4 indicate that only one HRV indicator value, pNN50, correlated significantly with $\mathrm{VO}_{2}$ max. When indicators were $\mathrm{HR}$ normalized by dividing by $\mathrm{RR}$ or $\mathrm{RR}^{2}$, no significant correlations were found. All significant correlation coefficients were between 0.19 and 0.41 indicating very low to moderate associations.

Simple linear regression analyses (Table 5) showed that only $\mathrm{HR}, \mathrm{RR}$, and pNN50 explained a significant $(p<0.05)$ proportion of the variation in $\mathrm{VO}_{2}$ max. Stepwise multiple regression (Table 5) indicated that, when including all nine indicators (HR, RR, RMSSD, pNN50, LF, HF, LF/HF, SD1, SD2), the statistical model containing a linear combination of HR and HF, explained the largest proportion of the variation, i.e., is the best predictor of variation in $\mathrm{VO}_{2} \max 20.1 \%$ ). However, $\mathrm{HR}$ entered the model first and by itself explains $17 \%$ of the variation, while the
Table 4 | Pearson's correlation coefficients ( $r$ ) and significance ( $p$-value) for $\mathrm{VO}_{2}$ max and $\mathrm{HRV}$ indicators.

\begin{tabular}{llll}
\hline $\begin{array}{l}\text { HRV } \\
\text { Indicators }\end{array}$ & $\begin{array}{l}\text { Indirect VO} \\
\boldsymbol{r}(\boldsymbol{p} \text {-value) }\end{array}$ & $\begin{array}{l}\text { Normalized HRV } \\
\text { indicators }\end{array}$ & $\begin{array}{l}\text { Indirect Vo } \mathbf{m a x} \\
\boldsymbol{r}(\boldsymbol{p} \text {-value) }\end{array}$ \\
\hline Mean RR & $0.41(<0.01)^{*}$ & $\mathrm{NA}$ & $\mathrm{NA}$ \\
Mean HR & $-0.41<0.01^{*}$ & $\mathrm{NA}$ & $\mathrm{NA}$ \\
RMSSD & $0.09(0.29)$ & $\mathrm{RMSSD} / \mathrm{RR}$ & $0.02(0.80)$ \\
pNN50 & $0.19(0.03)^{*}$ & $\mathrm{NA}$ & $\mathrm{NA}$ \\
LF & $0.10(0.21)$ & $\mathrm{LF} / \mathrm{RR}^{2}$ & $0.02(0.95)$ \\
HF & $0.001(0.99)$ & $\mathrm{HF} / \mathrm{RR}^{2}$ & $-0.03(0.72)$ \\
LF/HF & $0.02(0.82)$ & $\mathrm{NA}$ & $\mathrm{NA}$ \\
SD1 & $0.09(0.29)$ & $\mathrm{SD} 1 / \mathrm{RR}$ & $0.02(0.80)$ \\
SD2 & $0.14(0.10)$ & $\mathrm{SD} 2 / \mathrm{RR}$ & $0.02(0.79)$ \\
\hline
\end{tabular}

NA, Not applicable; ${ }^{*}$, significant: $p<0.05$.

Table 5 | Simple and stepwise multiple linear regression results.

\begin{tabular}{|c|c|c|c|}
\hline Regression type & $\begin{array}{l}\text { Predictors } \\
\text { of } \mathrm{VO}_{2} \mathrm{max} \\
\text { included }\end{array}$ & $\begin{array}{l}\text { Significant } \\
\text { predictors } \\
(p<0.05)\end{array}$ & $\begin{array}{l}\text { Coefficient of } \\
\text { determination } \mathbf{R}^{2}\end{array}$ \\
\hline Simple Regression & $\mathrm{HR}$ & HR & 0.170 \\
\hline Simple Regression & $\mathrm{RR}$ & $\mathrm{RR}$ & 0.161 \\
\hline Simple Regression & pNN50 & pNN50 & 0.035 \\
\hline Multiple Regression & $\begin{array}{l}\text { Nine indicators } \\
\text { HR, RR, RMSSD, } \\
\text { pNN50,LF, HF, } \\
\text { LF/HF, SD1, SD2 }\end{array}$ & $\mathrm{HR}$ and $\mathrm{HF}$ & 0.201 \\
\hline Multiple Regression & $\begin{array}{l}\text { HR and six } \\
\text { normalized HRV } \\
\text { indicators: } \\
\text { HR/RR, } \\
\text { RMSSD/RR, } \\
\text { LF/RR }^{2}, H F / R^{2} \text {, } \\
\text { LF/HF, SD1/RR, } \\
\text { SD2/RR }\end{array}$ & $\mathrm{HR}$ and $\mathrm{HF} / \mathrm{RR}^{2}$ & 0.190 \\
\hline
\end{tabular}


inclusion of HF added only an additional 3.1\% to the coefficient of determination. Stepwise multiple regression also showed that, when including $\mathrm{HR}$ and all normalized HRV indicators (RMSSD/RR, LF/RR $\left.{ }^{2}, \mathrm{HF} / \mathrm{RR}^{2}, \mathrm{LF} / \mathrm{HF}, \mathrm{SD} 1 / \mathrm{RR}, \mathrm{SD} 2 / \mathrm{RR}\right)$, the statistical model consisting of a linear combination of $\mathrm{HR}$ and $\mathrm{HF} / \mathrm{RR}^{2}$, will be the best predictor of variation in $\mathrm{VO}_{2} \mathrm{max}$, that is $19.0 \%$. HR on its own explained $16.5 \%$ and a combination of HR and $\mathrm{HF} / \mathrm{RR}^{2}$ thus, explained only a further $2.5 \%$.

\section{DISCUSSION}

The primary hypothesis was that straightforward measurements such as HR and RR intervals show stronger correlations with $\mathrm{VO}_{2}$ max than the HRV indicators, and is on their own stronger predictors of $\mathrm{VO}_{2}$ max than the more complex indicators of HRV. Study results partially confirmed the first hypotheses. Moderately strong correlations were found between $\mathrm{HR}$ and RR intervals vs. $\mathrm{VO}_{2}$ max. The only other correlation found (pNN50) was of very low strength $(r=0.19)$. The stepwise multiple regression analysis indicated that HR predicted $17 \%$, and the combination of $\mathrm{HR}$ and $\mathrm{HF}$ predicted $20.1 \%$ of the variation in $\mathrm{VO}_{2} \mathrm{max}$, when entering the following HRV indicators: HR, RR, RMSSD, pNN50, LF, HF, LF/HF, SD1, SD2. With regards to the second hypothesis multiple regression analysis showed that the combination of $\mathrm{HR}$ and $\mathrm{HF} / \mathrm{RR}^{2}$ explained only $2.5 \%$ more of the variation in $\mathrm{VO}_{2} \mathrm{max}$, than $\mathrm{HR}$ alone $(16.5 \%)$, when including all normalized indicators.

Recent publications by Sacha et al. (2013) suggested that, because of physiological and mathematical relationships between HR and HRV, the dependence of HRV indicator values on HR can be modified by mathematical manipulation. This is due to the known fact that RR variation is significant higher for a slow average HR than for higher mean HR. Following suggestions by Sacha et al. (2013) to minimize the HRV indicator's dependence on HR, correlational and regression statistical analyses in this study were performed firstly with the actual HR, RR, and HRV indicator values and again after each were normalized by dividing by the mean RR or $\mathrm{RR}^{2}$.

Correlations found between the HR, RR intervals, $\mathrm{pNN} 50$, and $\mathrm{VO}_{2}$ max were significant $(p<0.05)$, but of very low to medium strength $(0.19 \leq r \leq 0.41)$. A significant positive correlation was found between mean $\mathrm{RR}$ interval and $\mathrm{VO}_{2} \max (p<0.01 ; r=$ $0.41)$ and as expected a significant negative correlation between mean $\mathrm{HR}$ and $\mathrm{VO}_{2} \max (p<0.01 ; r=-0.41)$. These associations concur with some previous study findings. Higher fitness is associated with a lower $\mathrm{HR}$, higher $\mathrm{VO}_{2}$ max, and also higher vagal cardiac control (Nagai et al., 2004; Spierer et al., 2007; Gilder and Ramsbottom, 2008). The strength of the associations found in this study are lower than values reported in literature but this may be due to the fact that $\mathrm{VO}_{2}$ max was only indirectly determined and not directly with gas analyses.

The fact that only one HRV indicator ( $\mathrm{pNN} 50$ ) correlated significantly with $\mathrm{VO}_{2} \max$, may indicate that correlations between $\mathrm{HRV}$ indicators and $\mathrm{VO}_{2}$ max exist mainly due to the relationship between $\mathrm{HR}$ (independent of variability) and $\mathrm{VO}_{2}$ max.

Regression analysis confirmed that, when including $\mathrm{HR}, \mathrm{RR}$, RMSSD, pNN50, LF, HF, LF/HF, SD1, and SD2, the most important predictor of cardio vascular fitness and exercise ability as represented by $\mathrm{VO}_{2} \max$, is $\mathrm{HR}$. Results also showed when testing the normalized indicators, HR explained the largest percentage of the changes in $\mathrm{VO}_{2}$ max. Thus, $\mathrm{HR}$ on its own is the most important predictor of changes in an important cardiac health metric such as $\mathrm{VO}_{2}$ max. Thus, the more complex concept of HRV quantification unlock only a small percentage of additional information with regards to autonomic function, compared to information obtained from only HR and RR interval measurements. These results may indicate that during investigation of exercise ability $\left(\mathrm{VO}_{2} \mathrm{max}\right)$ phenomena, quantification of HRV may not add significant more value. This is a novel idea which should be investigated further in clinical populations.

Limitations of this study include the fact that $\mathrm{VO}_{2}$ max was not directly determined with gas analyses, but only indirectly determined. Another drawback is that the tachogram used to determine HRV, was sampled only in a resting, supine position. Previous studies indicated that HRV measured during a stressor, such as standing upright, show more significant correlations with cardiopulmonary fitness indicators than supine HRV measurements (Grant et al., 2009). For future studies it is recommended to investigate participants from other age groups as well as clinical groups to answer the question whether these results are only applicable to healthy young participants and if not; how it differs in other groups.

\section{REFERENCES}

Barakat, H., Barakat, H., and Baaj, M. K. (2012). CVD and obesity in transitional Syria: a perspective from the Middle East. Vasc. Health Risk Manag. 8, 145-150. doi: 10.2147/VHRM.S28691

Bravi, A., Longtin, A., and Seely, A. J. (2011). Review and classification of variability analysis techniques with clinical applications. Biomed. Eng. Online 10, 90. doi: 10.1186/1475-925X-10-90

Cooper, K. H. (1968). A means of assessing maximal oxygen intake. JAMA 203, 201-204. doi: 10.1001/jama.1968.03140030033008

Field, A. (1994). Discovering Statistics Using SPSS. London: Sage.

Gilder, M., and Ramsbottom, R. (2008). Change in heart rate variability following orthostasis relates to volume of exercise in healthy woman. Auton. Neurosci. 143, 73-76. doi: 10.1016/j.autneu.2008.06.002

Go, A. S., Mozaffarian, D., Roger, V. L., Benjamin, E. J., Berry, J. D., Borden, W. B., et al. (2013). Heart disease and stroke statistics update: a report from the American Heart Association. Circulation 127, e6-e245. doi: 10.1161/CIR.0b013e31828124ad

Grant, C. C., Clark, J. R., Janse van Rensburg, D. C., Strydom, N., and Viljoen, M. (2009). The relationship between exercise capacity and heart rate variability: supine and in response to an orthostatic stressor. Auton. Neurosci. 151, 186-188. doi: 10.1016/j.autneu.2009.08.015

Grant, C. C., Janse van Rensburg, D. C., Strydom, N., and Viljoen, M. (2011). Importance of tachogram length and period of recording during non-invasive investigation of the autonomic nervous system. Ann. Noninvasive Electrocardiol. 16, 131-139. doi: 10.1111/j.1542-474X.2011.00422.x

Kannel, W. B., Kannel, C., Paffenbarger, R. S. Jr., and Cupples, L. A. (1987). Heart rate and cardiovascular mortality: the Framingham study. Am. Heart J. 113, 1489-1494. doi: 10.1016/0002-8703(87)90666-1

Lahiri, M. K., Kannankeril, P. J., and Goldberger, J. J. (2008). Assessment of autonomic function in cardiovascular disease. J. Am. Coll. Cardiol. 51, 1725-1733. doi: 10.1016/j.jacc.2008.01.038

Landis, J. R., and Koch, G. G. (1977). The measurement of observer agreement for categorical data. Biometrics 33, 159-174. doi: 10.2307/2529310

Mourot, L., Bouhaddi, M., Perrey, S., Roillon, J. D., and Regnard, J. (2004). Quantitative Poincaré plot analysis of heart rate variability: effect of endurance training. Eur. J. Appl. Physiol. 91, 79-87. doi: 10.1007/s00421-0030917-0 
Nagai, N., Hamada, T., Kimura, T., and Moritani, T. (2004). Moderate physical exercise increases cardiac autonomic nervous system activity in children with low heart rate variability. Childs. Nerv. Syst. 20,209-214. doi: 10.1007/s00381-0040916-4.

Paterson, D. H., Govindasamy, D., Vidmar, M., Cunningham, D. A., and Koval, J. J. (2004). Longitudinal study of determinants of dependence in an elderly population. J. Am. Geriatr. Soc. 52, 1632-1638. doi: 10.1111/j.1532-5415.2004.52454.x

Sacha, J., Barabach, S., Statkiewicz-Barabach, G., Sacha, K., Muller, A., Piskorski, J., et al. (2013). How to strengthen or weaken HRV dependence on heart ratedescription of the method and its perspectives. Int. J. Cardiol. 168, 1660-1663. doi: 10.1016/j.ijcard.2013.03.038

Spierer, D. K., DeMeersman, R. E., Kleinveld, J., McPherson, E., Fullilove, R. E., Alba, A., et al. (2007). Exercise training improves cardiovascular and autonomic profiles in HIV. Clin. Auton. Res. 17, 341-348. doi: 10.1007/s10286-007-0441-0

Stringer, W. W. (2010). Cardiopulmonary exercise testing: current applications. Expert Rev. Respir. Med. 4, 179-188. doi: 10.1586/ers.10.8

Wilmore, J. H., and Costill, D. L. (2004). Physiology of sport and exercise. Hum. Kinet. 3, 140-142. doi: 10.1177/1356336X06069280

Yang, Q., Cogswell, M. E., Flanders, W. D., Hong, Y., Zhang, Z., Loustalot, F., et al. (2012). Trends in cardiovascular health metrics and associations with all-cause and CVD mortality among US adults. JAMA 307, 1273-1283. doi: 10.1001/jama.2012.339

Conflict of Interest Statement: The authors declare that the research was conducted in the absence of any commercial or financial relationships that could be construed as a potential conflict of interest.

Received: 27 August 2013; accepted: 01 November 2013; published online: 20 November 2013.

Citation: Grant CC, Murray C, Janse van Rensburg DC and Fletcher L (2013) A comparison between heart rate and heart rate variability as indicators of cardiac health and fitness. Front. Physiol. 4:337. doi: 10.3389/fphys.2013.00337

This article was submitted to Clinical and Translational Physiology, a section of the journal Frontiers in Physiology.

Copyright (C) 2013 Grant, Murray, Janse van Rensburg and Fletcher. This is an openaccess article distributed under the terms of the Creative Commons Attribution License (CC BY). The use, distribution or reproduction in other forums is permitted, provided the original author(s) or licensor are credited and that the original publication in this journal is cited, in accordance with accepted academic practice. No use, distribution or reproduction is permitted which does not comply with these terms. 